\title{
Mean Platelet Volume Predicts Severe COVID-19 Illness
}

\author{
Giuseppe Lippi, MD ${ }^{1}$ Brandon M. Henry, MD²,* \\ 1 Section of Clinical Biochemistry, University of Verona, Verona, Italy \\ ${ }^{2}$ Cardiac Intensive Care Unit, The Heart Institute, Cincinnati \\ Children's Hospital Medical Center, Cincinnati, Ohio \\ ${ }^{3}$ Department of Haematology, Sydney Centres for Thrombosis and \\ Haemostasis, Institute of Clinical Pathology and Medical Research \\ (ICPMR), NSW Health Pathology, Westmead Hospital, Westmead, \\ New South Wales, Australia
}

Semin Thromb Hemost 2021;47:456-459.

The parameter mean platelet volume (MPV) is a simple and relatively inexpensive laboratory measure that reliably reflects platelet size. Evidence has been provided that increased MPV values may be a hallmark of several thrombotic disorders, including acute coronary syndrome, stroke, venous thromboembolism, abdominal vein thrombosis, and even preeclampsia. ${ }^{1}$ Although the clinical significance of this laboratory parameter remains only partially unraveled, an enhanced MPV value mirrors the presence of large (mostly reticulated) platelets in the circulation, which are essentially hyperactivated and hyperreactive elements, thus justifying their frequent association with vascular thrombosis. ${ }^{2}$

Since coronavirus disease 2019 (COVID-19), a life-threatening infectious disease sustained by the severe respiratory syndrome coronavirus 2 (SARS-CoV-2), is frequently complicated by thrombotic episodes, both venous and arterial, ${ }^{3,4}$ we provide here an updated analysis of current scientific literature data exploring the association between MPV and illness severity in patients with COVID-19.

We conducted an electronic search in Scopus, MEDLINE (with PubMed interface), and the Web of Science, using the keywords "mean platelet volume" or "MPV" and "coronavirus disease 2019" or "COVID-19" in all fields, with no language or date restriction (i.e., up to February 18, 2021). Two authors analyzed the title, abstract and the full text of all items that could be identified according to the search criteria. Studies reporting MPV values in COVID-19 patients with or without severe illness were included in the pooled analysis. The full list of references of each article was also analyzed, with the purpose of identifying other eligible documents. The mean and standard deviation (SD) of MPV values were then in-

\footnotetext{
* These authors have equal senior authorship on this work.
}

published online April 26, 2021
Issue Theme Maintaining Hemostasis and Preventing Thrombosis in COVID-19 -Part II; Guest Editors: Emmanuel J. Favaloro, PhD, FFSc (RCPA) and Giuseppe Lippi, MD.

cluded in a pooled analysis, with calculation of weighted mean difference (WMD) and $95 \%$ confidence interval $(95 \% \mathrm{CI})$ of this parameter in patients with SARS-CoV-2 infection with or without severe disease. When mean value and SD were unavailable, these were extrapolated from available figures or calculated from sample size, median, and interquartile range, as proposed by Hozo et al. ${ }^{5}$ When multiple MPV measurements were presented, only values corresponding to peak severity were retained. A quality effects model was originally used, followed by a random effects model with the purpose of adjusting any heterogeneity that emerged among the various studies. The heterogeneity was assessed using $x^{2}$ test and $I^{2}$ statistic. The statistical analysis was performed with MetaXL, software version 5.3 (EpiGear International Pty Ltd., Sunrise Beach, Australia). The study was conducted in agreement with the declaration of Helsinki and within the terms of local legislation.

The original digital search enabled the identification of a total number of 83 documents. Following the elimination of duplicates across the three scientific databases, 65 were excluded as they did not specifically deal with COVID-19 ( $n=16)$, did not include MPV data $(n=13)$, or MPV values stratified for COVID-19 severity could not be retrieved or extrapolated $(n=17)$, were animal studies $(n=2)$, were review articles $(n=14)$ or editorial material $(n=2)$, or presented overlapping information already included in previous investigations $(n=1)$. No interreviewer disagreement emerged. A total number of 18 studies were finally included in our analysis, with 3,433 COVID-19 patients, 780 (22.7\%) with severe illness ( - Table $\mathbf{1}$ ). ${ }^{6-23}$ All except two were crosssectional studies, with the majority from China $(7 ; 39 \%)$, and used heterogeneous combination of clinical endpoints for defining severity of COVID-19 (44\% included respiratory

(c) 2021. Thieme. All rights reserved. Thieme Medical Publishers, Inc., 333 Seventh Avenue, 18th Floor, New York, NY 10001, USA
DOI https://doi.org/ $10.1055 / \mathrm{s}-0041-1727283$. ISSN 0094-6176. 
Table 1 Summary of clinical studies which have investigated MPV values in COVID-19 patients with or without severe illness

\begin{tabular}{|c|c|c|c|c|}
\hline Authors & Setting & Study design & Sample size & Endpoint \\
\hline Asan et $\mathrm{al}^{6}$ & Turkey & Cross-sectional & 695 (4\% severe) & ICU admission \\
\hline Barrett et $\mathrm{al}^{7}$ & US & Cross-sectional & 100 (32\% severe) & Thrombosis or death \\
\hline Comer et $\mathrm{al}^{8}$ & Ireland & Cross-sectional & 54 (63\% severe) & ICU admission \\
\hline Ding et $\mathrm{al}^{9}$ & China & Cross-sectional & 72 (21\% severe) & Respiratory failure and/or ICU admission \\
\hline Fois et $\mathrm{al}^{10}$ & Italy & Cross-sectional & 119 (24\% severe) & Death \\
\hline Giusti et al $^{11}$ & Italy & Cross-sectional & 209 (15\% severe) & Death \\
\hline Güçlü et $a^{12}$ & Turkey & Cross-sectional & 212 (62\% severe) & Respiratory failure \\
\hline Ko et $\mathrm{al}^{13}$ & Korea & Cross-sectional & 333 (43\% severe) & ICU admission \\
\hline Lanini et al ${ }^{14}$ & Italy & Longitudinal & 379 (11\% severe) & Death \\
\hline Lin et $\mathrm{al}^{15}$ & China & Cross-sectional & 68 (68\% severe) & Respiratory failure and/or ICU admission \\
\hline Mertoglu et al $^{16}$ & Turkey & Cross-sectional & 555 (4\% severe) & ICU admission \\
\hline Ouyang et al ${ }^{17}$ & China & Longitudinal & 107 (23\% severe) & Death \\
\hline Taha et al $^{18}$ & US & Cross-sectional & 81 ( $81 \%$ severe) & Acute kidney injury \\
\hline Taj et al $^{19}$ & Pakistan & Cross-sectional & 29 (28\% severe) & Respiratory failure \\
\hline Wang et $\mathrm{al}^{20}$ & China & Cross-sectional & 61 (39\% severe) & Respiratory failure and/or ICU admission \\
\hline Xing et $\mathrm{al}^{21}$ & China & Cross-sectional & 61 (13\% severe) & Respiratory failure and/or ICU admission and/or death \\
\hline Xiong et $\mathrm{al}^{22}$ & China & Cross-sectional & 57 (67\% severe) & Respiratory failure and/or ICU admission \\
\hline Zhang et $a^{23}$ & China & Cross-sectional & 241 (24\% severe) & Respiratory failure and/or ICU admission \\
\hline
\end{tabular}

Abbreviations: COVID-19, coronavirus disease 2019; ICU, intensive care unit; MPV, mean platelet volume.

failure and/or intensive care unit admission, 33\% death, and 6\% acute kidney failure or thrombosis; - Table $\mathbf{1}$ ).

The results of the pooled analysis of the 18 studies are shown in - Fig. 1. In all studies, the difference of MPV values between patients with or without severe COVID-19 illness was positive, reaching statistical significance in 14 of 18 (78\%). Overall, the WMD of MPV was 0.63 (95\% CI, 0.35-0.90) fL using a quality effects model (heterogeneity was high, $I^{2}=91 \%$ ), increasing to 0.74 (95\% CI, 0.51-0.97)
fL using a random effects model. MPV values were found to be increased by $6.3 \%$ (95\% CI, 3.6-9.0\%) in COVID-19 patients with adverse clinical outcomes compared with those without progression to severe disease. In a subanalysis of the four studies that included only mortality as clinical endpoint, the WMD of MPV values in random effects model further increased to 1.2 (95\% CI, 0.38-2.01) fL between patients who died and those who survived $\left(I^{2}=97 \%\right)^{10,11,14,17}$

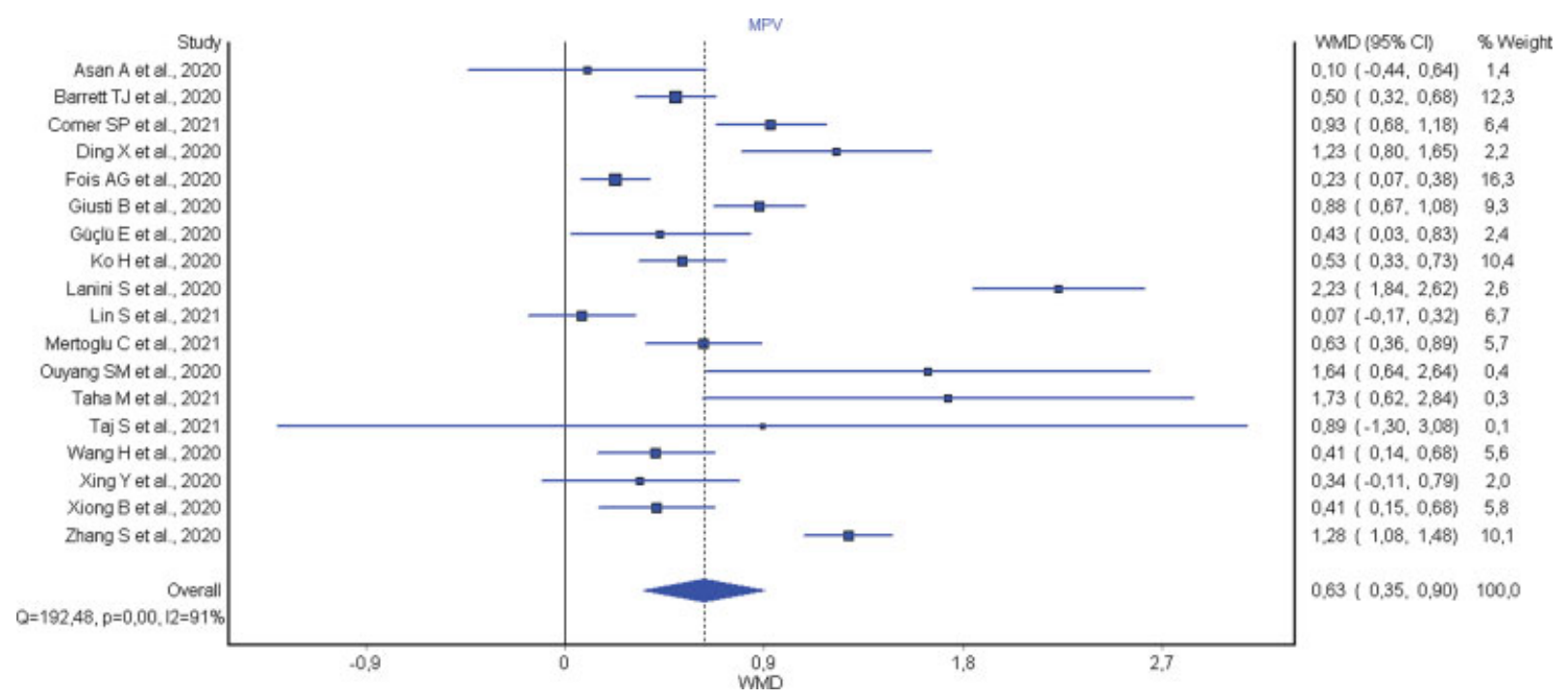

Fig. 1 Weighted mean difference (WMD) and 95\% confidence interval $(95 \% \mathrm{Cl})$ of mean platelet volume (MPV) in patients with coronavirus disease 2019 (COVID-19) with or without severe illness. 
The results of this pooled analysis of available scientific literature clearly attest that MPV, which reflects larger circulating platelets, may be a significant predictor of adverse clinical progression in patients with COVID-19, playing an even more important role in predicting mortality. This evidence is not unexpected, as platelet biology appears to be largely perturbed in patients with SARS-CoV-2 infection, and further contributes to magnify their risk of developing pulmonary or disseminated thrombosis. ${ }^{24,25}$ Although a thoughtful discussion on the interplay between platelets and SARS-CoV-2 infection has already been provided in some previous articles, ${ }^{24,25}$ a brief overview on the significance of these findings is perhaps advisable. SARS-CoV-2 can contribute to platelets activation via a variety of mechanisms and different pathways, such as direct binding to platelet surface, provoking endothelial injury, sustaining an inflammation-related release of von Willebrand factor (VWF) compounded by a concomitant decrease in the activity of ADAMTS-13 (a disintegrin and metalloproteinase with a thrombospondin type 1 motif, member 13 ) which would then lead to impaired cleavage of ultralarge VWF, as well as by reducing heparan sulfates at blood vessel surface and impairing generation of vasoeffective mediators (e.g., nitric oxide, prostacyclin, and prostaglandin). ${ }^{4}$ Platelet activation may also occur as a consequence of thrombin generation occurring after concomitant activation of blood coagulation. ${ }^{26}$ Moreover, direct SARS-CoV-2 and spike protein binding to platelet angiotensin-converting enzyme 2 (ACE2) has been shown to activate platelets in vitro, while spike has been shown to potentiate thrombus formation in vivo. Such SARS-CoV-2/spike to platelet ACE2 binding results in platelet stimulation, causing release of coagulation factors and inflammatory cytokines, with promotion of leukocyte-platelet aggregates formation. ${ }^{23}$ All the aforementioned mechanisms may hence synergistically act (or contribute) to generate hyperactive platelets, phenotypically recognizable by an increased size, which would then play a role to trigger the micro- and macrothrombotic events that are commonly seen in COVID-19 patients with severe or critical disease, ultimately fostering unfavorable prognosis, including an enhanced risk of death. ${ }^{27}$ Indirect support to this theory emerges from recent evidence that in-hospital aspirin ( $81 \mathrm{mg}$ daily) administration in COVID-19 patients has been associated with nearly $50 \%$ reduction in the risk of death. ${ }^{28}$

In conclusion, the results of our pooled analysis highlight that platelet volume is very frequently increased in COVID19 patients with severe illness, especially in those at higher risk of dying, such that routine measurement of MPV along with other useful laboratory parameters ${ }^{29,30}$ should be considered for identifying those cases at higher risk of adverse progression, needing more assiduous and even more aggressive care.

\section{Conflict of Interest}

None declared.

\section{References}

1 Lippi G, Sanchis-Gomar F, Favaloro EJ. Mean platelet volume in arterial and venous thrombotic disorders. J Lab Med 2020;44(05): 305-312

2 Chesnutt JK, Han HC. Platelet size and density affect shearinduced thrombus formation in tortuous arterioles. Phys Biol 2013;10(05):056003

3 Levi M, Thachil J. Coronavirus disease 2019 coagulopathy: disseminated intravascular coagulation and thrombotic microangiopathy-either, neither, or both. Semin Thromb Hemost 2020;46 (07):781-784

4 Lippi G, Sanchis-Gomar F, Favaloro EJ, Lavie CJ, Henry BM. Coronavirus disease 2019-associated coagulopathy. Mayo Clin Proc 2021;96(01):203-217

5 Hozo SP, Djulbegovic B, Hozo I. Estimating the mean and variance from the median, range, and the size of a sample. BMC Med Res Methodol 2005;5:13

6 Asan A, ÜstÜndaĞ Y, Koca N, et al. Do initial hematologic indices predict the severity of COVID-19 patients? Turk J Med Sci 2021;51 (01):39-44

7 Barrett TJ, Lee AH, Xia Y, et al. Platelet and vascular biomarkers associate with thrombosis and death in coronavirus disease. Circ Res 2020;127(07):945-947

8 Comer SP, Cullivan S, Szklanna PB, et al; COCOON Study Investigators. COVID-19 induces a hyperactive phenotype in circulating platelets. PLoS Biol 2021;19(02):e3001109

9 Ding X, Yu Y, Lu B, et al. Dynamic profile and clinical implications of hematological parameters in hospitalized patients with coronavirus disease 2019. Clin Chem Lab Med 2020;58(08): 1365-1371

10 Fois AG, Paliogiannis P, Scano V, et al. The systemic inflammation index on admission predicts in-hospital mortality in COVID-19 patients. Molecules 2020;25(23):5725

11 Giusti B, Gori AM, Alessi M, et al. SARS-CoV-2 induced coagulopathy and prognosis in hospitalized patients: a snapshot from Italy. Thromb Haemost 2020;120(08):1233-1236

12 Güçlü E, Kocayiğit H, Okan HD, et al. Effect of COVID-19 on platelet count and its indices. Rev Assoc Med Bras (1992) 2020;66(08): $1122-1127$

13 Ko H, Chung H, Kang WS, et al. An artificial intelligence model to predict the mortality of COVID-19 patients at hospital admission time using routine blood samples: development and validation of an ensemble model. J Med Internet Res 2020;22(12): e25442

14 Lanini S, Montaldo C, Nicastri E, et al. COVID-19 disease-temporal analyses of complete blood count parameters over course of illness, and relationship to patient demographics and management outcomes in survivors and non-survivors: a longitudinal descriptive cohort study. PLoS One 2020;15(12):e0244129

15 Lin S, Mao W, Zou Q, Lu S, Zheng S. Associations between hematological parameters and disease severity in patients with SARS-CoV-2 infection. J Clin Lab Anal 2021;35(01):e23604

16 Mertoglu C, Huyut MT, Arslan Y, Ceylan Y, Coban TA. How do routine laboratory tests change in coronavirus disease 2019? Scand J Clin Lab Invest 2021;81(01):24-33

17 Ouyang SM, Zhu HQ, Xie YN, et al. Temporal changes in laboratory markers of survivors and non-survivors of adult inpatients with COVID-19. BMC Infect Dis 2020;20(01):952

18 Taha M, Sano D, Hanoudi S, et al. Platelets and renal failure in the SARS-CoV-2 syndrome. Platelets 2021;32(01):130-137

19 Taj S, Kashif A, Arzinda Fatima S, Imran S, Lone A, Ahmed Q. Role of hematological parameters in the stratification of COVID-19 disease severity. Ann Med Surg (Lond) 2021;62:68-72

20 Wang H, Xing Y, Yao X, et al. Retrospective study of clinical features of COVID-19 in inpatients and their association with disease severity. Med Sci Monit 2020;26:e927674 
21 Xing Y, Wang H, Yao XH, et al. Analysis of factors for disease progression in 61 patients with COVID-19 in Xiaogan, Hubei, China. Eur Rev Med Pharmacol Sci 2020;24(23):12490-12499

22 Xiong B, Liu T, Luo P, et al. Prominent hypercoagulability associated with inflammatory state among cancer patients with SARSCoV-2 infection. Front Oncol 2020;10:1345

23 Zhang S, Liu Y, Wang X, et al. SARS-CoV-2 binds platelet ACE2 to enhance thrombosis in COVID-19. J Hematol Oncol 2020;13(01):120

24 Larsen JB, Pasalic L, Hvas AM. Platelets in coronavirus disease 2019. Semin Thromb Hemost 2020;46(07):823-825

25 Parra-Izquierdo I, Aslan JE. Perspectives on platelet heterogeneity and host immune response in coronavirus disease 2019 (COVID19). Semin Thromb Hemost 2020;46(07):826-830

26 Benati M, Salvagno GL, Nitto S, et al. Thrombin generation in patients with coronavirus disease 2019. Semin Thromb Hemost 2021 (epub ahead of print). Doi: 10.1055/s-0041-1722844
27 Malas MB, Naazie IN, Elsayed N, Mathlouthi A, Marmor R, Clary B. Thromboembolism risk of COVID-19 is high and associated with a higher risk of mortality: a systematic review and meta-analysis. EClinicalMedicine 2020;29:100639

28 Meizlish ML, Goshua G, Liu Y, et al. Intermediate-dose anticoagulation, aspirin, and in-hospital mortality in COVID-19: a propensity score-matched analysis. Am J Hematol 2021;96(04): 471-479

29 Favaloro EJ, Lippi G. Recommendations for minimal laboratory testing panels in patients with COVID-19: potential for prognostic monitoring. Semin Thromb Hemost 2020;46(03): 379-382

30 Christensen B, Favaloro EJ, Lippi G, Van Cott EM. Hematology laboratory abnormalities in patients with coronavirus disease 2019 (COVID-19). Semin Thromb Hemost 2020;46(07): 845-849 